\title{
可重复使用记忆介质上可存储的最 大信息量
}

\author{
符方伟 \\ (南开大学数学系, 天津 300071)
}

\section{关键词可直复使用记忆介质、多用户信息论、容贯区域、信息贯}

Heegard $^{[1]}$, Ahlswede 和 Simonyi ${ }^{[2]}$ 提出了具有随机干扰和差错的可重复使用记忆介质的 一般数学模型. 他们用一个概率信道来描述记忆介质上每一个存储元的状态变化机制, 然后 采用多用户信息论的方法来研究可重复使用记忆介质的编码问题和容量问题. 当我们在信息 存储介质上多次存取信息时, 对应于编、译码者知道或不知道记个介质的状态, 我们有四种 编、译码情形: (1) 编、译码者都知道记忆介质的状态, (2) 编码者知道, 译码者不知道, (3) 编 码者不知道, 译码者知道, (4) 编、译码者都不知道. Ahlswede 和 Simonyi 在文献[2] 中研究了 (3), (4) 情形下可重复使用记忆介质的容量问题. Heegard 在文献[1] 的第三部分研究了情形 (2)下可重复使用记忆介质的容量问题,给出了可重复使用记讴介质上可存储的最大信息量 的一个下界. 本文证明这个界是紧的, 从而完全确定了情形 (2)下可重复使用记忆介质上可 存储的最大信息量.

\section{1 数学模型和主要结果}

$\mathscr{X}, \mathscr{Y}$ 是两个有限集合, $\mathscr{X}$ 称为输人字母表, $\mathscr{Y}$ 称为输出字母表, 同时也是状态字母表. $Q=\{q(y): y \in \mathscr{Y}\}$ 为 $\mathscr{Y}$ 上一个概率分布, 称为初始状态分布. $W=\{W(y \mid x, s): x \in \mathscr{X}, s \in \mathscr{Y}, y \in \mathscr{Y}\}$ 为 $\mathscr{X} \times \mathscr{Y}$ 到 $\mathscr{Y}$ 上一个条件概率分布.

设一个可重复使用记忆介质由 $n$ 个存储元组成, 它的初始状态为 $s=\left(s_{1}, s_{2}, \cdots, s_{n}\right) \in \mathscr{O}^{n}$ 的 概率为 $\prod_{i=1}^{n} q\left(s_{i}\right)$. 当我们在这个记忆介质上多次存取信息时, 如果这个记忆介质的状态为 $s=\left(s_{1}\right.$, $\left.s_{2}, \cdots, s_{n}\right) \in \mathscr{Y}^{n}$, 输人元为 $x=\left(x_{1}, x_{2}, \cdots, x_{n}\right) \in \mathscr{C}^{n}$, 则这个记忆介质的输出元为 $y=\left(y_{1}, y_{2}, \cdots, y_{n}\right) \in$ $\mathscr{Y}^{n}$ 的概率为 $\prod_{i=1}^{n} W\left(y_{i} \mid x_{i}, s_{i}\right)$. 同时 $y$ 成为记忆介质新的状态. 假设我们要在这个记忆介质上连 续存取 $T$ 次信息.

在编、译码情形 (2) 下, 一个 $\left(n, M_{1}, M_{2} \cdots, M_{T}\right)$ 码为 $T$ 对编、译码函数 $\left\{f_{t}, g_{t}\right\}_{t=1}^{T}$. 设 $I_{t}=$ $\left\{1,2, \cdots, M_{t}\right\}, f_{t}: I_{t} \times \mathscr{Y}^{n} \rightarrow \mathscr{C}^{n}, g_{t}: \mathscr{Y}^{n} \rightarrow I_{t}, t=1,2, \cdots T$. 设 $Y^{n}(0)$ 为初始状态随机变量,

1994-01-29 收稿.

*国家教委优秀青年教师基金资助项目. 
概率分布为 $P_{r}\left\{Y^{n}(0)=s\right\}=\prod_{i=1}^{n} q\left(s_{i}\right), s=\left(s_{1}, s_{2}, \cdots, s_{n}\right) \in \mathscr{Y}^{n}$. 设 $V_{1}, V_{2}, \cdots V_{T}$ 为分另取值于 $I_{1}, I_{2}$, $\cdots, I_{T}$ 上相互独立且服从均匀分布的随机变量. 它们分别为第 $1,2, \cdots, T$ 次存取信息时存储 的消息元. 在第 $t$ 次存取信息时, $Y^{n}(t-1)$ 为记忆介质的状态随机变量, $X^{n}(t)=f_{t}\left(V_{t}, Y^{n}(t-1)\right)$ 为记忆介质的输人随机变量, 通过条件概率分布 $W$ 产生记忆介质的输出随机变量 $Y^{n}(t)$, 同 时 $Y^{n}(t)$ 也成为第 $t+1$ 次存取信息时记忆介质的状态随机变量. 令 $\bar{V}_{t}=g_{t}\left(Y^{n}(t)\right)$, 称为消息 $V_{t}$ 的复制元. 令 $P_{e}(t)=P_{r}\left\{V_{t} \neq \bar{V}_{t}\right\}, t=1,2, \cdots, T$. 则这个码的误差概率定义为 $P_{e}=\max _{1 \leqslant t \leqslant T} P_{e}(t)$.

我们用 $H(\cdot)$ 表示随机变量的嫡, $H(\cdot \mid \cdot)$ 表示条件嫡, $I(\cdot ; \cdot)$ 表示互信息, $88_{+}$表示非 负实数集合. $h(x)=-x \log x-(1-x) \log (1-x)$ 表示二元摘函数.

向量 $\left(R_{1}, R_{2}, \cdots, R_{T}\right) \in F_{+}^{T}$ 称为可达的, 如果对任意的 $\varepsilon>0$, 存在一个 $\left(n, M_{1}, M_{2}, \cdots\right.$, $M_{T}$ ) 码, 使得 $R_{t}=\frac{1}{n} \log M_{t}, t=1,2, \cdots, T$, 且 $P_{e}<\varepsilon$. 所有可达向量构成的集合称为这个可重

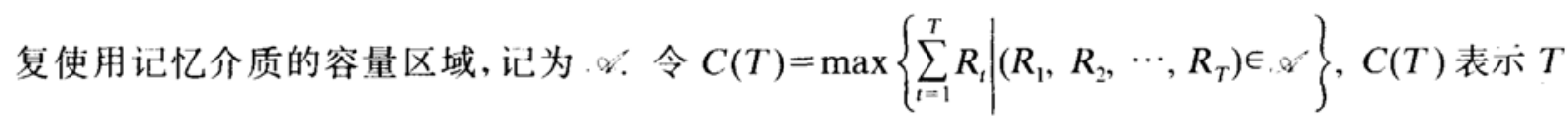
次存取信息过程中, 平均矮个存储元可存储的最大信息量.

设 $\mathscr{H}_{1}, \mathscr{H}_{2}, \cdots, \mathscr{Y}_{T}$ 为 $T$ 个有限集, $P_{t}=\left\{P_{t}\left(u_{t}, x_{t} \mid y_{t-1}\right): u_{t} \in \mathscr{H}_{t}, x_{t} \in \mathscr{X}^{\prime}, y_{t-1} \in \mathscr{Y}\right\}, t=1,2, \cdots, T$ 为条件概率分布. 我们可以构造一组随机变量 $\left(Y_{0}, U_{1}, X_{1}, Y_{1}, U_{2}, X_{2}, Y_{2}, \cdots, U_{T}, X_{T}, Y_{T}\right)$, 联合概率分布为 $q\left(y_{0}\right) P_{1}\left(u_{1}, x_{1} \mid y_{0}\right) W\left(y_{1} \mid x_{1}, y_{0}\right) P_{2}\left(u_{2}, x_{2} \mid y_{1}\right) W\left(y_{2} \mid x_{2}, y_{1}\right) \cdots P_{T}\left(u_{T}, x_{T} \mid y_{T-1}\right) W\left(y_{T} \mid x_{T}\right.$, $y_{T}$, ). 实际上这里 $U_{1}, U_{2}, \cdots, U_{T}$ 为辅助随机变量, 在多用户信息论中我们经常引人辅助随 机变量去刻划容量区域. 令 $B=\left\{\left(R_{1}, R_{2}, \cdots, R_{T}\right) \in{ }_{+}^{T} \mid\right.$ 存在一组随机变量 $\left(Y_{0}, U_{1}, X_{1}, Y_{1}, U_{2}\right.$, $\left.X_{2}, Y_{2}, \cdots, U_{T}, X_{T}, Y_{T}\right)$, 具有上述联合概率分布形式, 使得

$$
\left.R_{t} \leqslant I\left(U_{t} ; Y_{t}\right)-I\left(U_{t} ; Y_{t-1}\right), t=1,2, \cdots, T\right\},
$$

设. $\mathscr{B}$ 为 $B$ 产生的闭凸包, 即 $\not s=\overline{\operatorname{conv}}\{B\}$. 令 $D(T)=\max \left\{\sum_{t=1}^{T} R_{t} \mid\left(R_{1}, R_{2}, \cdots, R_{T}\right) \in \mathscr{B}\right\}$. Heegard

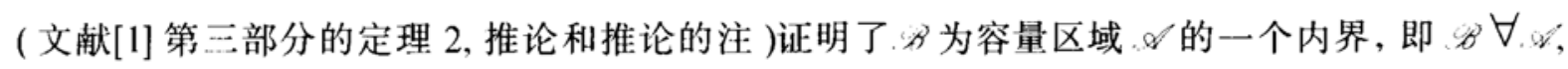
从而i $C(T) \geqslant D(T)$. 本文的主要结果为

\section{定理 $1 \quad C(T)=D(T)$.}

注 定理 1 确定了可重复使用记忆介质上可存储的最大信息量, 但是仍然没有能够确 定容量区域, 一些信息论学者猜想作实际上给出了容量区域, 即 $x^{\prime}$. 本文的结果似乎使 我们更有理由相信这个猜想, 但到H前为止, 还没有人能够确定容量区域的表达形式.

\section{2 定 理 的 证 明}

我们只要证明逆编码定理: 如果向量 $\left(R_{1}, R_{2}, \cdots, R_{T}\right)$ 可达, 则 $\sum_{t=1}^{T} R_{t} \leqslant D(T)$.

因为间量 $\left(R_{1}, R_{2}, \cdots, R_{T}\right)$ 可达, 则存在本文第一部分所叙述的编、译码函数和相应的随 机变量.由 Fano 不等式知

$$
H\left(V_{t} \mid Y^{n}(t)\right) \leqslant H\left(V_{t} \mid g_{t}\left(Y^{n}(t)\right)\right)=H\left(V_{t} \mid \bar{V}_{t}\right)
$$




$$
\begin{aligned}
\leqslant P_{e}(t) \log M_{t} & +h\left(P_{e}(t)\right) \leqslant n \varepsilon R_{t}+h(\varepsilon), \\
I\left(V_{t} ; Y^{n}(t)\right) & =H\left(V_{t}\right)-H\left(V_{t} \mid Y^{n}(t)\right) \\
& =n R_{t}-H\left(V_{t} \mid Y^{n}(t)\right) .
\end{aligned}
$$

由 (1) 和 (2) 式, 且 $V$, 与 $Y^{n}(t-1)$ 独立, 我们有

$$
\begin{aligned}
n R_{t}(1-\varepsilon)-h(\varepsilon) & \leqslant I\left(V_{t} ; Y^{n}(t)\right) \\
& =I\left(V_{t} ; Y^{n}(t)\right)-I\left(V_{t}, Y^{n}(t-1)\right) \\
& =H\left(Y^{n}(t)\right)-H\left(Y^{n}(t-1)\right)-\left[H\left(V_{t}, Y^{n}(t)\right)-H\left(V_{t}, Y^{n}(t-1)\right)\right], \\
(1-\varepsilon) \sum_{t=1}^{T} R_{t}-\frac{T}{n} h(\varepsilon) \leqslant & \frac{1}{n} \sum_{t=1}^{T}\left[H\left(Y^{n}(t)\right)-H\left(Y^{n}(t-1)\right)\right] \\
& \quad-\frac{1}{n} \sum_{t=1}^{T}\left[H\left(V_{t}, Y^{n}(t)\right)-H\left(V_{t}, Y^{n}(t-1)\right)\right] .
\end{aligned}
$$

$Y^{n}(T)=\left(Y_{1}(T), Y_{2}(T), \cdots, Y_{n}(T)\right)$ 满足 $H\left(Y^{n}(T)\right) \leqslant \sum_{i=1}^{n} H\left(Y_{i}(T)\right), Y^{n}(0)=\left(Y_{1}(0), \quad Y_{2}(0), \quad Y_{n}(0)\right)$ 的 各分量相互独立, 则 $H\left(Y^{n}(0)\right)=\sum_{i=1}^{n} H\left(Y_{i}(0)\right)$, 故

$$
\begin{aligned}
\sum_{t=1}^{T}\left[H\left(Y^{n}(t)\right)-H\left(Y^{n}(t-1)\right)\right] & =H\left(Y^{n}(T)\right)-H\left(Y^{n}(0)\right) \\
& \leqslant \sum_{i=1}^{n}\left[H\left(Y_{i}(T)\right)-H\left(Y_{i}(0)\right)\right] \\
& =\sum_{i=1}^{n} \sum_{i=1}^{T}\left[H\left(Y_{i}(t)\right)-H\left(Y_{i}(t-1)\right)\right] .
\end{aligned}
$$

因定 $t$, 设 $Y^{n}(t)=\left(Y_{1}(t), Y_{2}(t), \cdots, Y_{n}(t)\right), Y^{n}(t-1)=\left(Y_{1}(t-1), Y_{2}(t-1), \cdots, Y_{n}(t-1)\right)$, 令 $Z_{i}(t)$ $=\left(V_{t}, Y_{1}(t), Y_{2}(t), \cdots, Y_{i}(t), Y_{i+1}(t-1), Y_{i+2}(t-1), \cdots, Y_{n}(t-1)\right), i=0,1, \cdots, n$. 显然 $Z_{0}(t)=$ $\left(V_{t}, Y^{n}(t-1)\right), Z_{n}(t)=\left(V_{t}, Y^{n}(t)\right)$, 令 $U_{i}(t)=\left(V_{t}, Y_{1}(t), Y_{2}(t), \cdots, Y_{i-1}(t), Y_{i+1}(t-1), Y_{i+2}(t-1)\right.$, $\left.\cdots, Y_{n}(t-1)\right)$, 显然 $Z_{i}(t)=\left(U_{i}(t), Y_{i}(t)\right), Z_{i-1}(t)=\left(U_{i}(t), Y_{i}(t-1)\right)$.

$$
\begin{aligned}
H\left(V_{t}, Y^{n}(t)\right)-H\left(V_{t}, Y^{n}(t-1)\right) & =H\left(Z_{n}(t)\right)-H\left(Z_{0}(t)\right) \\
& =\sum_{i=1}^{n}\left[H\left(Z_{i}(t)\right)-H\left(Z_{i-1}(t)\right)\right] \\
& =\sum_{i=1}^{n}\left[H\left(U_{i}(t), Y_{i}(t)\right)-H\left(U_{i}(t), Y_{i}(t-1)\right)\right] .
\end{aligned}
$$

由 (4), (5), (6) 式知

$$
\begin{aligned}
(1-\varepsilon) \sum_{t=1}^{T} R_{t}-\frac{T}{n} h(\varepsilon) \leqslant & \frac{1}{n} \sum_{i=1}^{n} \sum_{t=1}^{T}\left[H\left(Y_{i}(t)\right)-H\left(Y_{i}(t-1)\right)\right. \\
& \left.-H\left(U_{i}(t), Y_{i}(t)\right)+H\left(U_{i}(t), Y_{i}(t-1)\right)\right] \\
= & \frac{1}{n} \sum_{i=1}^{n} \sum_{t=1}^{T}\left[I\left(U_{i}(t) ; Y_{i}(t)\right)-I\left(U_{i}(t) ; Y_{i}(t-1)\right)\right] .
\end{aligned}
$$

因定 $i$, 我们有随机变量 $\left(Y_{i}(0), U_{i}(1), X_{i}(1), Y_{i}(1), U_{i}(2), X_{i}(2), Y_{i}(2), \cdots, U_{i}(T), X_{i}(T), Y_{i}\right.$ $(T)$ ), 令 
$P_{t}^{(i)}\left(u_{t}, x_{t} \mid y_{t-1}\right)=P_{r}\left\{U_{i}(t)=u_{t}, X_{i}(t)=x_{t} \mid Y_{i}(t-1)=y_{t-1}\right\}, t=1,2, \cdots T$. 取一组新的随机变 量 $\left(\bar{Y}_{i}(0), \bar{U}_{i}(1), \bar{X}_{i}(1), \bar{Y}_{i}(1), \bar{U}_{i}(2), \bar{X}_{i}(2), \bar{Y}_{i}(2), \cdots, \bar{U}_{i}(T), \bar{X}_{i}(T), \bar{Y}_{i}(T)\right)$, 其联合概率分布 为 $q\left(y_{0}\right) P_{1}^{(i)}\left(u_{1}, x_{1} \mid y_{0}\right) W\left(y_{1} \mid x_{1}, y_{0}\right) P_{2}^{(i)}\left(u_{2}, x_{2} \mid y_{1}\right) W\left(y_{2} \mid x_{2}, y_{1}\right) \cdots P_{T}^{(i)}\left(u_{T}, x_{T} \mid y_{T-1}\right) W\left(y_{T} \mid x_{T}, y_{T-1}\right)$. 通过简 单的概率计算易知对 $t=1,2, \cdots, T$, 随机变量 $\left(\bar{Y}_{i}(t-1), \bar{U}_{i}(t), \bar{X}_{i}(t), \bar{Y}_{i}(t)\right)$ 与 $\left(Y_{i}(t-1), U_{j}(t)\right.$, $\left.X_{i}(t), Y_{i}(t)\right)$ 同分布. 故 $I\left(U_{i}(t) ; Y_{i}(t)\right)=I\left(\bar{U}_{i}(t) ; \bar{Y}_{i}(t)\right), I\left(U_{i}(t) ; Y_{i}(t-1)\right)=I\left(\bar{U}_{i}(t) ; \bar{Y}_{i}(t-1)\right)$. 则

$$
\begin{gathered}
\sum_{t=1}^{T}\left[I\left(U_{i}(t) ; Y_{i}(t)\right)-I\left(U_{i}(t) ; Y_{i}(t-1)\right)\right] . \\
=\sum_{i=1}^{T}\left[I\left(\bar{U}_{i}(t) ; \bar{Y}_{i}(t)\right)-I\left(\bar{U}_{i}(t) ; \bar{Y}_{i}(t-1)\right)\right] \leqslant D(T) .
\end{gathered}
$$

由 (7) 和 (8) 式知

$$
(1-\varepsilon) \sum_{t=1}^{T} R_{t}-\frac{T}{n} h(\varepsilon) \leqslant D(T)
$$

令 $\varepsilon \rightarrow 0$ 知 $\sum_{t=1}^{T} R_{t} \leqslant D(T)$.

注 定理的证明过程不能推导出容量区域 $\mathscr{A}=\mathscr{B}$, 这是因为当 $t=2,3, \cdots, T$ 时, $Y^{n}(t-1)$ 的分量不一定相互独立.

致谢 作者感谢沈世镥教授, R. Ahlswede 教授和 C. Heegard 教授在本文写作过程中所 给予的指导和帮助.

\section{参考文 献}

(1) Heegard, C., IEEE Trans. Inform. Theory, 1985, IT-31(1): 34--42.

[2] Ahlswede, R., Simonyi, G., IEEE Trans. Inform. Theory, 1991, IT-37(4): 1143-1150. 